\title{
Experience of exercise in people with severe haemophilia: A qualitative study
}

By Stephanie Taylor ${ }^{1,2}$, Francine Toye ${ }^{1}$, Karen Barker ${ }^{1,3}$

The benefits of regular activity and exercise are well established, yet a third of the population of the European Union does not achieve the recommended levels of activity. For patients with haemophilia, some activity can cause bleeding into joints, leading to potential joint damage, whereas other activities can protect the joint from bleeds and further destruction. This study investigates the understanding and experience of exercise and activity in people with haemophilia (PWH). We conducted semi-structured interviews with six men with severe haemophilia using a consecutive sampling framework and Interpretative Phenomenological Analysis (IPA). We identified five themes:

- "I don't think about haemophilia, I've just got to deal with it" A level of acceptance of awareness of their condition, but they do not want it to rule their lives.

- "I don't let my limitations hold me back" Striving to find activities they can participate in, despite joint impairment.

- "The worst thing anyone can do is stop being active" Belief that activity helps to strengthen joints, gives confidence and improves both body and mind.

- "The best thing they did was to not wrap me up in cotton wool" Knowledge of haemophilia, how to treat and recognise bleeds, and finding activities to suit their bodies.

STEPHANIE TAYLOR

Physiotherapy Research Unit, Nuffield Orthopaedic Centre and Oxford Haemophilia and Thrombosis Centre, Oxford University Hospitals NHS Foundation Trust, Windmill Road, Oxford, OX3 7LE, UK

FRANCINE TOYE

Physiotherapy Research Unit, Nuffield Orthopaedic Centre, Oxford University Hospitals NHS Foundation Trust, Windmill Road, Oxford, OX3 7LD, UK

\section{KAREN BARKER}

Physiotherapy Research Unit, Nuffield Orthopaedic Centre and Nuffield Department of Orthopaedics, Rheumatology and Musculoskeletal Sciences, University of Oxford, Windmill Road, OX3 7HE, UK

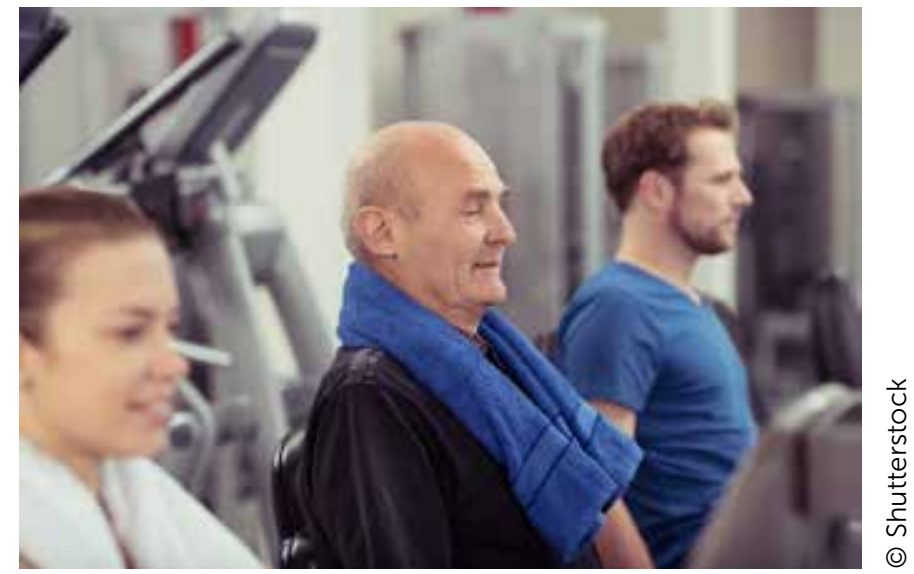

- "Time constraints at home" Common barriers to exercise, as in the general population.

Our findings provide clinicians with insight into understanding the barriers to exercise and activity in men with severe haemophilia. This can help clinicians to offer the most appropriate support and allow PWH to find an activity or exercise that suits them. Our findings demonstrate that even those with severe haemophilia wish to remain active and do not want to let their limitations prevent them from exercising. Findings also indicate that being given the education and freedom to make independent decisions about exercise and activities are valued. This suggests that clinicians need to provide a therapeutic environment where their patients can feel safe to make sensible choices about types and level of activity.

Keywords: Haemophilia, Exercise, Activity, Qualitative study

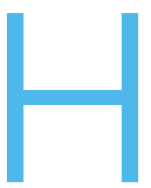

aemophilia is an X-linked bleeding disorder characterised by deficiencies of Factor VIII or IX. With the advent of safe clotting factor preparations, most patients have a near-normal life expectancy, with almost half of patients in the UK over the age of 18 years ${ }^{[1,2]}$. 
Frequent musculoskeletal bleeds in people with severe haemophilia $(\mathrm{PWH})$ can cause limitations and deficiencies of the musculoskeletal system, affecting the performance of daily living activities as compared with healthy individuals ${ }^{[3,4]}$.

The benefits of regular activity and exercise are well established, yet a third of the population of the European Union does not achieve the recommended levels of activity - a major issue with our everincreasing sedentary behaviour ${ }^{[5]}$. PWH have a relatively sedentary lifestyle. Sherlock and colleagues found that severity of haemophilia and age were the main indicators for reduced activity ${ }^{[6]}$. PWH have lower muscle strength and anaerobic power compared with age-matched controls, and their lower rate of physical activity is a risk factor for low bone mineral density and osteoporosis ${ }^{[7,8]}$. Because of the frequent musculoskeletal bleeds, PWH may limit their physical activities early in life, thus developing physiological, neurological and functional disorders as a consequence of their sedentary lifestyle ${ }^{[6]}$. Historically, PWH were unable to participate in many sports and physical activities due to their low factor levels; indeed, until the early 1970s, physical activity was contra-indicated as an "unnecessary" risk ${ }^{[9,10]}$ With recent advances in pharmacological treatment, PWH can now participate in regular exercise with protection against bleeds. However, although new products have revolutionised treatment and improved quality of life for PWH, treatment does not offer full protection - joint bleeds may still occur, despite regular prophylaxis.

Debate as to what are the best forms of exercise for $\mathrm{PWH}$ is ongoing, but there is consensus that exercise helps in improving muscle strength, joint stability and joint proprioception, in turn helping to reduce joint bleeds and subsequent haemophilic arthropathy ${ }^{[11-16]}$. Though exercise, PWH could benefit from improvement in muscle strength, balance and proprioception capacity ${ }^{[16-18]}$. Furthermore, acute exercise sessions increment levels of Factor VIII, subsequently improving coagulation in mild patients ${ }^{[19]}$. Thus, current advice strongly recommends physical activity and seeks to avoid the vicious cycle of inactivity and the sequelae of musculoskeletal complications ${ }^{[9,20-23]}$

There is a paucity of qualitative studies though which to gain insight into personal views and experiences of PWH on physical activity. If we are to motivate $\mathrm{PWH}$ to be increasingly physically active, it is important for the multidisciplinary team (MDT) to understand patients' beliefs and experiences of being physically active with haemophilia. With this insight, we can then aim to deliver focused advice and patient centred care. To address this gap in the literature, this study aims to investigate the beliefs and experiences of PWH on being active and participating in exercise.

\section{Methods}

A qualitative study using an interpretive phenomenological analysis (IPA) approach ${ }^{[24]}$. Ethical approval for the study was granted by NRES committee (ref no: 15/LO/1095).

We selected a consecutive sample of patients, aged 18-59, with a diagnosis of severe haemophilia as indicated by factor level. Participants followed a range of prophylactic regimes and one had inhibitors. Table 1 gives details of the patients' age and prophylaxis regime. All participants spoke English as their first language.

We contacted all adult patients with severe haemophilia registered at the Oxford Haemophilia and Thrombosis Centre. Ages ranged from 18-74 years old. Patients were invited by post to take part in the study to explore their understanding and attitudes about exercise and activity. Of 110 invitations sent, 31 gave consent for further contact from the study team. Six consecutive participants were recruited and interviewed. The interviews were reviewed until it was felt that useful ideas had developed and the data added no further insight. As IPA aims to explore a detailed interpretative account of a small sample, six subjects were considered a sufficient number ${ }^{[24]}$.

A preliminary semi-structured interview guide

Table 1: Description of participants' treatment regimen

\begin{tabular}{l|l|l|l|} 
AGE IN YEARS & PSEUDONYM & PROPHYLAXIS REGIME & INHIBITOR STATUS \\
\hline 46 & Ian & Treated every 3 days & No Inhibitor \\
\hline 22 & Joe & On demand & Inhibitor \\
\hline 18 & Andrew & Daily prophylaxis & No Inhibitor \\
\hline 59 & John & On demand & No Inhibitor \\
\hline 58 & Simon & Alternate day prophylaxis & No Inhibitor \\
\hline 43 & Jim & 3 times a week prophylaxis & No Inhibitor \\
\hline
\end{tabular}


Table 2: Themes of questions for interview

Experiences of being active with haemophilia; examples of activity undertaken

Any barriers and benefits of exercise/physical activity; eg mental health, pain, increased strength

Participant's history of being physically active, from childhood, in school, being a teenager and adulthood. Experiences of others around them towards exercise/ activity, eg family, friends, teachers, work colleagues.

How would the participant encourage someone to be more active?

was developed (Table 2). Patients were invited to be interviewed at a place and time of their choice, and travel expenses were offered. All participants chose to be interviewed at the hospital. A senior physiotherapist with nine years' experience in haemophilia, interviewed all participants. She made every attempt to put participants at ease and to assure them that the interview would not affect their ongoing treatment. The physiotherapist is a clinician, not a qualitative researcher, and was supported by a social scientist with more than 20 years' experience in qualitative research. Interviews were digitally audio-recorded and transcribed verbatim. Interviews lasted from 32-60 minutes (average 42 minutes). Field notes, memos and a reflexive diary were recorded throughout.

\section{Data Analysis}

Audio recordings were listened to and transcripts read until they become familiar. Data was coded in accordance with Interpretative Phenomenological Analysis (IPA), which is concerned with understanding the meaning attributed to a particular experience or phenomenon ${ }^{[25]}$. The transcripts were coded and broken into discrete units and re-coded. Codes found to be conceptually similar were grouped under a list of emergent themes, with the aim of developing connections between the data. Concerted efforts were made to remain close to the data and to continually explore meaning. Further strategies to promote rigour, including peer review, code-recode audits, constant comparison of data, codes and categories occurred throughout the analysis. FT assisted in the peer review of emerging codes and categories, and independently coded a sample of the interview data. Each interviewee was given a pseudonym for presentation purposes to preserve anonymity.

\section{Results}

Five main themes were identified:

- "I don't think about haemophilia, I've just got to deal with it"

- "I don't let my limitations hold me back"

- "The worst thing anyone can do is stop being active"

- "The best thing they did was to not wrap me up in cotton wool"

- "Time constraints at home"

These themes are described and illustrated below with narrative examples.

"I don't think about haemophilia, I've just got to deal with it"

There was a consensus among participants that they got on with their lives, did not give haemophilia much thought and did not let it stop them exercising.

"I don't consider myself a haemophiliac until | need to treat my joints for any reason. So, I don't go walking around with that consciously in my head, but subconsciously it's always there." lan, aged 46

Although all participants expressed frustration with the bleeds into their joints, they all described it as something that they just had to deal with.

"Obviously, there are times if I get a bleed it's pretty demoralising and stuff like that. But I try and do succeed, I think, in everyday life in not letting it be a major part of my life, cos it's not a conscious thing, it's something I've done. And if I did think about it the whole time and let it dictate things, then it would just bring you down, which I strongly don't think should happen." Joe, aged 22

The frustration focused on having to be still and wait for bleeds to subside.

"I hate having a bleed, having to be still. I don't like sitting at home, as you have to rest it. It is frustrating." Andrew, aged 18

"I don't let my limitations hold me back" All participants felt that it was important to be able to adapt as their bodies changed with age and ability, and to be positive about what they can do, rather than to focus on what they cannot. Some embraced new sports or activities.

"It sort of came to a point of trying to find things I could do - not so much trying to find things I couldn't do but things I could do." Joe, aged 22

"I am a positive person, so I embraced cycling and jumped into that, trying to allow that to be the focal point, rather than the unhappiness of not being able to play golf." lan, aged 46 
When certain activities became difficult, participants described how they adapted so that they could continue.

"I say kick a ball - I will throw it and my son kicks it back." Jim, aged 44

"The worst thing anyone can do is stop being active" Activity was viewed as a good thing, as it helped with joint strength and gave participants confidence in their body.

"Exercise gives you confidence, so sometimes what holds me back is just a lack of confidence. I think, 'If I do this I feel my knee is going to go,' whereas actually doing that and being out cycling not only gives you the confidence, but afterwards your legs feel a bit stronger. It gives you the confidence to do something similar or more next time." Joe, aged 22

Exercise was described as helping with social inclusion, and preventing feelings of isolation and being different.

"I think everyone should be as active as possible keeps you stronger. I think you have less bleeds and socially you can join in with everyone and don't feel excluded." Andrew, aged 18

Participants expressed the importance of finding an activity that will take you through life; one that you will always be able to participate in and enjoy.

"I would say it's to find activities that will not give you long-term effect on joints, cos once you have got a damaged joint that is just painful, long-term pain, that is no fun for anyone." Ian, aged 46

Participants felt that not all activity would suit everybody, although football was the only sport specifically mentioned that might potentially damage the joints. Participants had to balance the benefits of enjoying a sport with the trade-off of subsequent joint damage.

"We tried to ignore the condition we had and it gave us lots of problems as a consequence. Half my problems with my ankles was because I played football and shouldn't have done." John, aged 59

Some described how they were able to vary the amount and level of football they played to allow them to continue something they loved to participate in.

"I think that the ankle problems, as such, were when I was doing too much. But since I have been sensible with what I do, it is all fine now." Andrew aged 18

Others said that if they had been warned of the potential damage from football, they would have not played it as a child.
"If I was really given the choice now, I wouldn't have kicked a ball if I could have healthy ankles now and play sport that I can play into my old age!" Ian, aged 46

\section{"The best thing they did was to not wrap me up in cotton wool"}

Participants described how being trusted by their parents and clinicians was important. It was not useful to be "wrapped up in cotton wool". Letting boys explore activities for themselves and to participate in sports and exercise allowed them to learn what was achievable.

"I consider myself very lucky that my parents were very free with me and my brother and allowed us to be active as we would like and deal with the situations as they come, rather than wrapping us up in cotton wool, as that would have changed our lives considerably for the worse, I think." lan, aged 46

At school, the importance of being included and given the option to do alternative activities, rather than being singled out and excluded, was described.

"I think [it was] the teachers who made me feel different and I suppose it was them being nervous and stuff like that, but at the time I didn't like that. And I am sure now I would probably have sympathy with them, but when you are growing up you don't want to be treated differently. So, the best teachers were the ones who were aware and you could talk to but treated you like normal." Simon, aged 58

Participants felt that education was essential to be able to manage the condition independently.

"I really can't think of much my parents did stop me doing. I think the best thing they did was educate me in a way so I know what to look out for or what to do. But when it came down to it, they were quite happy to let me get on and get on with it, which I think is really important - for which I am incredibly grateful." Joe, aged 22

\section{"Time constraints at home"}

Participants reported that it was not always easy to fit activity and exercise into hectic life schedules. These time constraints were independent of their haemophilia.

"Time constraints at home and finding a nice place to cycle. Like being near a nice path rather than on an A road. It would be nice to just cycle from the house. I can't complain, but the hassle of putting the bikes on the car... It is just another barrier." Jim, aged 44 


\section{Discussion}

The haemophilia population are now living longer, thanks to modern management, and are also more active than previous generations of PWH. Activity/ exercise is now advocated for this population, and with individualised prophylaxis many $\mathrm{PWH}$ can participate in most types of exercise. Understanding the experience of being active with haemophilia is important for clinicians to help them advise and educate PWH about the most suitable form of activity. Exercise is advocated for $\mathrm{PWH}$, but the type of exercise needs to be individualised and assessed for risk, biomechanical demands and the individual's physical capacity ${ }^{[26] .}$

Many papers have discussed types of exercise and exercise prescription, but the number of studies looking into patients' experiences of being active with haemophilia is limited ${ }^{[27]}$. Czepa et al. found that PWH evaluated their physical performance lower than that of healthy age-matched controls ${ }^{[11]}$. Other studies have focused on the psychosocial aspects of haemophilia, looking at areas such as quality of life, psychological wellbeing, coping strategies and family issues with haemophilia ${ }^{[28-30]}$. Cassis performed a systematic review of recent studies into psychosocial aspects of haemophilia and found limited useful evidence in only 24 studies included for review; 19 used validated generic questionnaires, together with disease-specific questionnaires, e.g. Haemo-QoL-A [27]. Only a few studies interviewed patients directly and none, to our knowledge, asked specifically about exercise and activity.

Our findings were in agreement with other studies showing that people with chronic health conditions report a positive quality of life. Participants expressed a feeling of getting on with life by not focusing on their haemophilia and not allowing themselves to be defined by their condition. They tended to have a strong internal locus of control, and these favourable psychological characteristics appeared to reduce the perceived seriousness of haemophilia ${ }^{[28,30]}$. All participants wanted to be seen and treated like everyone else. The adoption of positive coping strategies and the positive outlook reported by participants in our study may help to explain their generally good experiences with activity and exercise. They expressed frustration at having bleeds, but accepted that this was part of life and something that was not going to get them down. This acceptance of their condition resonates with other qualitative studies of haemophilia ${ }^{[28]}$.
Activity was reported as important to all, which is encouraging, as there is much in the haemophilia literature about the importance of exercise in joint protection and general body health, both physical and psychological. Subjects expressed the confidence that exercise gave them and that it enabled social inclusion. Despite some physical limitations, the drive to find an activity to take part in was expressed by all participants.

Football was the only sport mentioned that had detrimental effects. While it gave the participants great pleasure, some blamed football for their joint damage. Others stated that if they had been warned about the potential effect of the bleeds on their joints, they would have chosen a different sport. Fromme et al. reported on sports participation in $\mathrm{PWH}$, but did not find such a strong correlation with football, instead reporting a range of sports associated with complications ${ }^{[31]}$. However, they reported that $11.4 \%$ of respondents named football as the sport they would most like to be able to participate in. In the Netherlands, $77 \%$ stated that they especially regret not being allowed to play football, reflecting the dominance of football as the team sport of choice in Europe ${ }^{[32]}$.

Our findings showed that, unsurprisingly, $\mathrm{PWH}$ experienced many of the same barriers to exercise as those in the general population: lack of interest, and inability to find the time and opportunity to exercise. These findings are supported by Sherlock, who reported that pain and arthritis prevented participation in sport by $\mathrm{PWH}$, but also lack of interest and time ${ }^{[6]}$. Having knowledge about haemophilia, and the tools to make decisions about activity and exercise, were expressed as very important. The multidisciplinary team and parents were viewed as important in helping inform, facilitate changes and educate ${ }^{[21,27]}$. Furthermore, we found that there was a need for trust between PWH and those giving them advice about activity. Choice of activity was important, rather than a focus on only the activities that are not advised, alongside more positive promotion of those that are more suitable.

Limitations of the study include that the decision to focus on persons with severe haemophilia. We did not explore the experiences of persons with moderate or mild haemophilia. Due to the nature of the recruitment process, only those persons happy to consent to talk about their experiences were included. This may affect the findings, as this group demonstrated characteristics of having a positive outlook and motivation to be active and altruistic. The results may not be transferrable across the population of those with severe haemophilia. 


\section{Conclusion}

To our knowledge, this is the first study interviewing people with haemophilia about their experiences and attitudes towards exercise and activity. While there are many studies that have investigated quality of life and the impact of haemophilia, these articles tend to use validated questionnaires with fixed questions. Although their outcome is interesting, it does not adequately explore the real experiences of $\mathrm{PWH}$.

Our study confirms the findings of others that having an inherited condition does not always cause a poor quality of life. We found that the PWH interviewed accept their condition, are positive towards exercise and find it of benefit, both physically and psychologically. They are adaptable and can alter activities as required as their bodies change. Finally, $\mathrm{PWH}$ are no different from the rest of the population with regard to some of the barriers to activity.

\section{Acknowledgments}

All authors contributed to the design and coordination of the study. ST carried out the interviews, led the data analysis and drafted the initial manuscript. FT independently coded transcripts and contributed to the data analysis. KB peer reviewed the data analysis, drafted and revised the manuscript, and was responsible for the study's research governance. All authors read and approved the final manuscript.

\section{Disclosures}

The authors have advised no interests that might be perceived as posing a conflict or bias. This is an Open Access article distributed under the terms of the Creative Commons Attribution License (http:// creativecommons.org/licenses/by/2.0), which permits unrestricted use, distribution, and reproduction in any medium, provided the original work is properly cited.

\section{References}

1. Tagliaferri A. Awaiting evidence-based recommendations on prophylaxis in adult patients. Haemophilia 2010; 16: 955-6.

2. UKHCDO. Bleeding disorder statistics for April 2014 to March 2015. A report from the National Haemophlia Database. 2015. Available from http://www.ukhcdo.org/wp-content/ uploads/2016/02/Bleeding-Disorder-Statistics-for-April-2014to-March-2015-v2-for-website.pdf (accessed 14 December 2016)

3. Buzzard B. Physiotherapy, rehabilitation and sports in countries with limited replacement coagulation factor supply. Haemophilia 2007; 13: 44-6.

4. Hilberg T, Czepa D, Freialdenhoven D, Boettger MK. Joint pain in people with hemophilia depends on joint status. Pain 2011;
152: 2029-35.

5. WHO. Physical activity fact sheet. 2016. Available from wWW who.int/mediacentre/factssheets/fs385/en/ (accessed 14 Decembe 2016).

6. Sherlock E, O'Donnell J, White B, Blake C. Physical activity levels and participation in sport in Irish people with haemophilia. Haemophilia 2010; 16: e202-e9.

7. Al-Sharif FA-G, Al-Jiffri OH, El-Kader SMA, Ashmawy EM. Impact of mild versus moderate intensity aerobic walking exercise training on markers of bone metabolism and hand grip strength in moderate hemophilia A patients. African Health Sciences 2014; 14: 11-6.

8. Kempton C, Antoniucci D, Rodriguez-Merchan E. Bone health in persons with haemophilia. Haemophilia 2015; 21: 568-77.

9. Negrier C, Seuser A, Forsyth A, et al. The benefits of exercise for patients with haemophilia and recommendations for safe and effective physical activity. Haemophilia 2013; 19: 487-98.

10. Hilberg T, Herbsleb M, Puta C, Gabriel H, Schramm W. Physical training increases isometric muscular strength and proprioceptive performance in haemophilic subjects. Haemophilia 2003; 9: 86-93.

11. Czepa D, Von Mackensen S, Hilberg T. Haemophilia \& Exercise Project (HEP): subjective and objective physical performance in adult haemophilia patients - results of a cross-sectional study. Haemophilia 2012; 18: 80-5.

12. Groen W, Van der Net J, Bos K, et al. Joint health and functional ability in children with haemophilia who receive intensive replacement therapy. Haemophilia 2011; 17: 783-90

13. González L, Peiro-Velert C, Devis-Devis J, et al. Comparison of physical activity and sedentary behaviours between young haemophilia A patients and healthy adolescents. Haemophilia 2011; 17: 676-82

14. Fearn M, Hill K, Williams $S$, et al. Balance dysfunction in adults with haemophilia. Haemophilia 2010; 16: 606-14.

15. Hill K, Fearn M, Williams S, et al. Effectiveness of a balance training home exercise programme for adults with haemophilia: a pilot study. Haemophilia 2010; 16: 162-9.

16. Herbsleb M, Hilberg T. Maximal and submaximal endurance performance in adults with severe haemophilia. Haemophilia 2009; 15: 114-21.

17. El-Sayed MS, Ali ZE-S, Ahmadizad S. Exercise and training effects on blood haemostasis in health and disease. Sports Med 2004; 34: 181-200.

18. Harris S, Boggio L. Exercise may decrease further destruction in the adult haemophilic joint. Haemophilia 2006; 12: 237-40.

19. Koch B, Luban NL, Galioto FM, et al. Changes in coagulation parameters with exercise in patients with classic hemophilia. Am J Hematol 1984; 16: 227-33.

20. Pelletier JR, Findley TW, Gemma SA. Isometric exercise for an individual with hemophilic arthropathy. Phys Ther 1987; 67: 1359-64.

21. Wittmeier K, Mulder K. Enhancing lifestyle for individuals with haemophilia through physical activity and exercise: the role of physiotherapy. Haemophilia 2007; 13: 31-7.

22. Blamey G, Forsyth A, Zourikian N, et al. Comprehensive elements of a physiotherapy exercise programme in haemophilia - a global perspective. Haemophilia 2010; 16 : 136-45.

23. Souza J, Simoes H, Campbell CG, et al. Haemophilia and 
exercise. Int J Sports Med 2012; 33: 83-8.

24. Smith JA, Osborn M. Interpretative Phenomenological Analysis. 2nd Ed Qualitative Psychology A practical guide to research methods. 2nd ed. London: SAGE Publications Ltd, 2003.

25. Smith JA, Flowers P, Larkin M. Interpretative

Phenomenological Analysis. Theory, Method and Research. London: SAGE Publications Ltd; 2009.

26. Gomis M, Querol F, Gallach J, González L, Aznar J. Exercise and sport in the treatment of haemophilic patients: a systematic review. Haemophilia 2009; 15: 43-54.

27. Cassis FY, Querol F, Forsyth A, lorio A. Psychosocial aspects of haemophilia: a systematic review of methodologies and findings. Haemophilia 2012; 18: e101-e14.

28. Beeton K, Neal D, Lee C. An exploration of health-related quality of life in adults with haemophilia - a qualitative perspective. Haemophilia 2005; 11: 123-32.

29. Barlow J, Stapley J, Ellard D, Gilchrist M. Information and selfmanagement needs of people living with bleeding disorders: a survey. Haemophilia 2007; 13: 264-70.

30. Triemstra AH, Van der Ploeg HM, Smit C, et al. Well-being of haemophilia patients: a model for direct and indirect effects of medical parameters on the physical and psychosocial functioning. Soc Sci Med 1998; 47 (5): 581-93.

31. Fromme A, Dreeskamp K, Pollmann $\mathrm{H}$, et al. Participation in sports and physical activity of haemophilia patients. Haemophilia 2007; 13: 323-7.

32. Schoenmakers MA, Gulmans VA, Helders PJ, van den Berg HM. Motor performance and disability in Dutch children with haemophilia: a comparison with their healthy peers. Haemophilia 20017 (3): 293-8.

\section{The Journal of Haemophilia Practice}

\section{An open-access journal for sharing experience in the care of people with bleeding disorders}

\section{www.haemjournal.com}

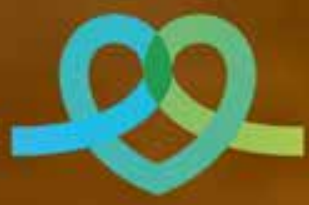

